As another test, values of $y$ were taken every $10^{\circ}$ from the equation

$$
y=1000 \sin x+333 \sin 3 x\left(x-10^{\circ}\right)+100 \sin 5\left(x-15^{\circ}\right)+50 \sin 13 x .
$$

Corresponding values of $x$, determined by the above rule, varied not more than a sixth of one per cent. from the values determined by tables. For curves even more irregular, it is believed the method will give results sufficiently accurate for most purposes and usually more accurate than can be otherwise obtained. For a fuller discussion, references and a comparison with other methods, see a paper about to be published by the authors entitled "Derivation of Wave-form of Flux from Wave-form of Electromotive Force," Electric Journal, I9I 5 .

CORNELL UNIVERSITY.

\title{
The Electric Resolution of the Series Lines in the Mercury SPECTRUM. ${ }^{1}$
}

By Georg Wendt and Reinhard A. Wetzel.

$\mathrm{T}^{\mathrm{H}}$

$\mathrm{HE}$ transverse Stark effect was obtained in canal rays with electric fields measuring from 50,000 to 90,000 volts per centimeter.

In the sharp subordinate series of mercury triplets the following lines were investigated: $\lambda 2,925 ; 2,894 ; 2,752 ; 2,576 ; 2,464 \AA$. The photograms obtained show no appreciable splitting or displacement of the lines with these fields under the dispersion at our disposal.

In the diffuse subordinate series of mercury triplets we investigated the following: $\lambda_{3}, \mathrm{I}_{32} ; 3,024 ; 2,967 ; 2,803 ; 2,699 ; 2,652 ; 2,534 ; 2,482 \AA$. With the exception of $\lambda 2,967$ all show splitting and displacements due to the action of the electric field. Quantitatively the effect is not the same upon each member of the triplet. It is largest upon the first, smaller upon the second, and smallest upon the third component of the triplet. The effect also increases rapidly with the increasing term numbers of the series.

Results.-(I) A confirmation of Stark's series laws for the electric effectviz., there is a difference between sharp and diffuse subordinate series, there is an increase of the effect with increase of term number; (2) A confirmation of the fact that for the different elements the nature and magnitude of the electric effect upon series lines of the same term number is not a function of their atomic weights but first and foremost a characteristic of the chemical element (its valence electrons). The photograms show this fact even more beautifully than those obtained by Stark and Kirschbaum.

The above investigation was made at the Physical Institute of the Technical High School at Aachen, Germany, during July, I9I4. Thanks are due Prof. Stark for the privileges of the laboratory. His rich experience and thoughtful suggestion also served us during the entire work.

${ }^{1}$ Abstract of a paper presented at the New York meeting of the Physical Society, October 3I, I9I4. 